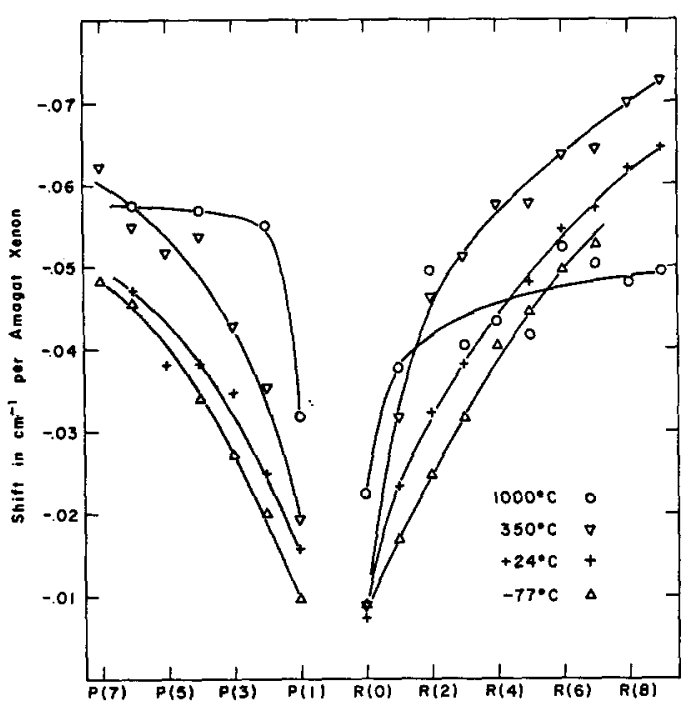

FIG. 1. Pressure-induced shifts of lines in the 2-0 band of $\mathrm{HCl}$ due to xenon; at four temperatures.

cell length was $30 \mathrm{~cm}$ for the observations at $-77^{\circ} \mathrm{C}$ and $50 \mathrm{~cm}$ for the others. The procedure for measuring the shifts was similar to that already described. ${ }^{3}$ The reference cell contained a small quantity of $\mathrm{HCl}$ only, and was at room temperature. The results are presented in the figure as shifts for a constant density of xenon.

In impact theories, ${ }^{4}$ collisions with an impact parameter smaller than a certain critical value $b_{0}$ are treated as "strong." While affecting linewidth, these strong collisions do not contribute to the shift.

If it is assumed that the interaction energy is proportional to the inverse sixth power of the intermolecular distance, impact theories suggest that for lines with small $J$ numbers, for which $b_{0}$ can be shown to be large, the shift should be proportional to $T^{3 / 10}$.

For high values of $J, b_{0}$ can be shown to be smaller, and a statistical factor arising from the intermolecular potential may play an important role. This factor favors closer collisions, leading to larger shifts, but since it decreases in importance with increasing temperature, it may be the cause of the reversal, at high $J$ numbers, of the direction of the temperature dependence that seems to be apparent in Fig. 1.

${ }^{1}$ M. A. Hirshfeld, J. H. Jaffe, and S. Kimel, J. Chem. Phys. 32, 297 (1960); D. H. Rank, W. B. Birtley, D. P. Eastman, and T. A. Wiggins, ibid. 32, 296 (1960); 33, 323 (1960). A. BenReuven, S. Kimel, M. A. Hirshfeld, and J. H. Jaffe, ibid. 35, 955 (1961).

Papers read by D. H. Rank and by S. Kimel at the International Conference on Spectral Line Shape and Molecular Interactions Rehovoth, August 1961.

2 Papers read by H. Margenau, by R. Herman, by A. D. Buckingham, ${ }^{1}$ by $R$. Englman, and $A$. Ben-Reuven at the Rehovoth Conference.

${ }^{3}$ S. Kimel, M. A. Hirshfeld, and J. H. Jaffe, J. Chem. Phys. 31, 81 (1959).

4. W. Anderson, Phys. Revs. 76, 647 (1949); C. J. Tsao and B. Curnutte, Geophysical Research Paper No. 69 (U. S. Air Force Cambridge Research Lab.) 1960.

\section{Evaluation of the Intermolecular Potential Well Depth from Observations of Rainbow Scattering: $\mathrm{Cs}-\mathrm{Hg}$ and $\mathrm{K}-\mathrm{Hg}^{*}$}

\author{
Fred A. Morse, $\dagger$ R. B. Bernstein, and H. U. Hostettler $\ddagger$ \\ Chemistry Department, University of Michigan, \\ Ann Arbor, Michigan \\ (Received January 17, 1962)
}

$\mathbf{T}$ THE present communication reports measurements of rainbow scattering for the $\mathrm{Cs}-\mathrm{Hg}$ and $\mathrm{K}-\mathrm{Hg}$ systems, from which the depths of the intermolecular potentials $\epsilon_{12}$ may be directly evaluated.

Mason $^{1}$ has presented a classical theoretical analysis of molecular beam scattering in which he pointed out the possibility of observing a discontinuity in the angular distribution at a critical deflection angle $\chi_{s}$, since termed ${ }^{2}$ the rainbow angle $\theta_{r}$. This angle corresponds to the trajectory exhibiting maximum attraction between the interacting particles, i.e., $\theta_{r}$ is the minimum of the deflection function $\theta\left(b^{*}\right)$. Since $\theta_{r}$ is strongly dependent upon the reduced relative collision energy $K=\frac{1}{2} \mu v_{r}{ }^{2} / \epsilon_{12}$, its location may be used to determine $\epsilon_{12}$ directly.

The experiments were carried out using velocity selected alkali beams scattered by a crossed, collimated $\mathrm{Hg}$ beam. The apparatus has been briefly described ${ }^{3}$; the velocity resolution of the selector was $4.7 \%$; only a small additional spread in relative speed $v_{r}$ is introduced by the thermal distribution in the $\mathrm{Hg}$ beam. The scattering angles and intensities have been corrected to the center of mass (c.m.) system, taking cognizance of the (average) $\mathrm{Hg}$ speed. Absolute cross sections

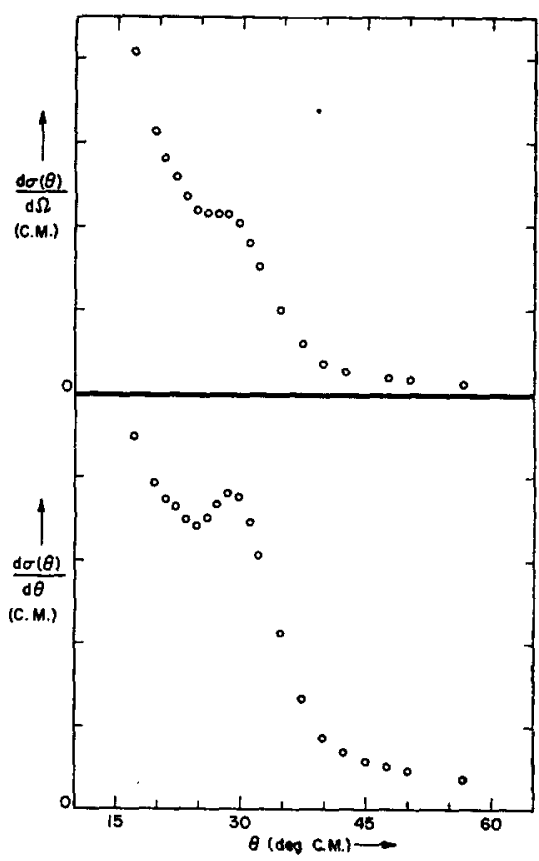

(a)

(b)

FIG. 1. (a). Example of rainbow scattering (observations corrected to the c.m. system. $\mathrm{K}-\mathrm{Hg}, v_{r}=1026 \mathrm{~m} / \mathrm{sec}$ ). (b) Alternate representation of the same data (see text). 


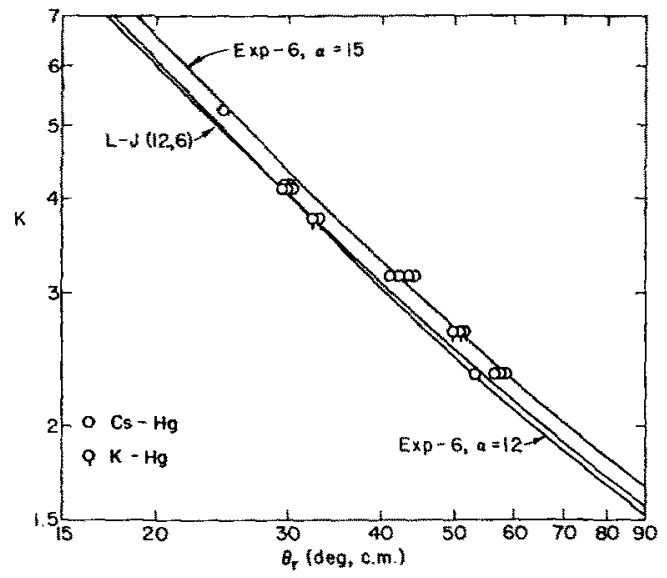

FIG. 2. Energy dependence of $\theta_{r}$. Solid lines: calculated, for indicated potential functions. Points: experimental, assuming $\epsilon / k=555^{\circ} \mathrm{K}$.

are not required for the present application; thus the vertical scales in Fig. 1 are arbitrary.

Figure 1(a) is an example of the differential elastic solid angle scattering cross section $d \sigma(\theta) / d \Omega$, for $\mathrm{K}-\mathrm{Hg}$ at $v_{r}=1026 \mathrm{~m} / \mathrm{sec}$, while Fig. 1(b) shows the corresponding differential polar scattering cross section $d \sigma(\theta) / d \theta=2 \pi \sin \theta d \sigma(\theta) / d \Omega$; analogous results ${ }^{4}$ were obtained for Cs-Hg. $\theta_{\text {r }}$ was taken to be the inflection point on the rapidly falling portion of $d \sigma(\theta) / d \Omega$ after the "bump."

Figure 2 shows calculated curves of $\theta_{r}$ vs $K$ for the Lennard-Jones $(12,6)^{5}$ and the exp- $6^{1}$ potential functions. The experimental points are superimposed; the ordinates have been fitted assuming $\epsilon_{12} / k=555^{\circ} \mathrm{K}$ for both the $\mathrm{Cs}-\mathrm{Hg}$ and $\mathrm{K}-\mathrm{Hg}$ systems. Over the range studied, a given fractional uncertainty in $\theta_{r}$ leads to nearly the same fractional error in the derived $\epsilon_{12}$. It can also be seen (Fig. 2) that the value obtained for $\epsilon_{12}$ is quite insensitive to the steepness assumed for the repulsion.

Without velocity selection the Maxwellian angular distributions show no evidence of rainbow scattering and indeed, over the angular range of the present study $\left(5-80^{\circ}\right.$ C.M. $)$, may be fitted with an extremely wide range of $\epsilon_{12}$ values. $^{8}$

* Financial assistance from the U. S. Atomic Energy Commission, Division of Research, is acknowledged.

$\dagger$ H. Riggs and E. L. Macauley Fellow, Rackham Graduate School.

$\$$ Now at Physikalisches Institut der Universität Freiburg, Switzerland.

E. A. Mason, J. Chem. Phys. 26, 667 (1957).

${ }^{2} \mathrm{~K}$. W. Ford and J. A. Wheeler, Ann. Phys. (N. Y.) 7, 287 (1959).

${ }^{3}$ H. U. Hostettler and R. B. Bernstein, Phys. Rev. Letters 5 , $318(1960)$.

- Rainbow scattering has also been observed by D. Beck, E. F. Greene, and J. Ross for $\mathrm{K}-\mathrm{Kr}$ and $\mathrm{K}-\mathrm{HBr}[\mathrm{E}, \mathrm{F}$. Greene (private communication)].

J. O. Hirschfelder, C. F. Curtiss, and R. B. Bird, Molecular Theory of Gases and Liguids (John Wiley \& Sons, Inc., New York, 1954).

${ }^{B}$ cf. H. Pauly, Z. Naturforsch. 14a, 1083 (1959).

\section{Comments and Errata}

\section{Erratum: Normal Vibrations of the Poly- mer Molecules of Helical Configuration. II. A Simple Method of Factoring of the Secular Equation}

\author{
[J. Chem. Phys. 35, 1050 (1961)] \\ Hiroyuki Tadokoro \\ Department of Polymer Science, Faculty of Science, \\ Osaka University, Nakanoshima, Kitaku, Osaka, Japan
}

IN Eq. (9), page 1052, for " $\sin 3 k \omega "$ in the matrix elements of the 1st row 3rd column, 2nd row 4th column, 3 rd row 1 st column, and 4 th row 2 nd column, read " $\sin 2 k \omega$ ".

\section{Notes}

\section{Observations in Proton NMR Spectra of Long-Range Coupling Between Protons and Carbon-13 in Natural Abundance}

\author{
D. R. MCADAMS \\ Esso Research Laboratories, Humble Oil \& Refining Company, \\ Baton Rouge, Louisiand
}

(Received November 17, 1961)

$\mathbf{T}$ HE effects of short-range coupling between protons and carbon-13 have been observable with available equipment for a number of years. The long-range coupling can now be observed in many compounds despite the difficulties imposed (1) by the low (about 1.1\%) natural abundance of $\mathrm{C}^{13}$, and (2) by the rather low coupling constants of 5-10 cps. This coupling may be measured directly in the proton spectrum whenever the principal central peak of the uncoupled protons is suffciently narrow (see Table I) of the order of $1 \frac{1}{2} \mathrm{cps}$. These observations were made with an unmodified A-60 NMR spectrometer manufactured by Varian Associates with sample spinning frequencies of about $35 \mathrm{cps}$.

TABI.E I. Observations on long-range coupling between protons and carbon- 13 in natural abundance.

\begin{tabular}{lll}
\hline \multicolumn{1}{c}{ Compound } & Proton species & $J$ (long $\left.\mathrm{C}^{13}-\mathrm{H}^{1}\right)$ \\
\hline acetonitrile & & $9.8-10.0$ \\
sodium acetate & & $5.8-6.0$ \\
methyl acetate & acetate & $6.8-6.9$ \\
acetophenone & methyl & 5.7 \\
acetaldehyde & methyl & 6.0 \\
toluene & methyl & 5 (broad) \\
\hline
\end{tabular}

